\title{
Revisiting the mouse model of oxygen-induced retinopathy
}

This article was published in the following Dove Press journal:

Eye and Brain

20 May 2016

Number of times this article has been viewed

\author{
Clifford B Kim ${ }^{1,2}$ \\ Patricia A D'Amore ${ }^{2-4}$ \\ Kip M Connor ${ }^{1,2}$ \\ 'Angiogenesis Laboratory, \\ Massachusetts Eye and Ear, \\ ${ }^{2}$ Department of Ophthalmology, \\ Harvard Medical School, ${ }^{3}$ Schepens \\ Eye Research Institute, Massachusetts \\ Eye and Ear, ${ }^{4}$ Department of \\ Pathology, Harvard Medical School, \\ Boston, MA, USA
}

\begin{abstract}
Abnormal blood vessel growth in the retina is a hallmark of many retinal diseases, such as retinopathy of prematurity (ROP), proliferative diabetic retinopathy, and the wet form of age-related macular degeneration. In particular, ROP has been an important health concern for physicians since the advent of routine supplemental oxygen therapy for premature neonates more than 70 years ago. Since then, researchers have explored several animal models to better understand ROP and retinal vascular development. Of these models, the mouse model of oxygeninduced retinopathy (OIR) has become the most widely used, and has played a pivotal role in our understanding of retinal angiogenesis and ocular immunology, as well as in the development of groundbreaking therapeutics such as anti-vascular endothelial growth factor injections for wet age-related macular degeneration. Numerous refinements to the model have been made since its inception in the 1950s, and technological advancements have expanded the use of the model across multiple scientific fields. In this review, we explore the historical developments that have led to the mouse OIR model utilized today, essential concepts of OIR, limitations of the model, and a representative selection of key findings from OIR, with particular emphasis on current research progress.
\end{abstract}

Keywords: ROP, OIR, angiogenesis

\section{History of the mouse model of OIR Initial clinical observations}

The first case reports of retrolental fibroplasia, now known as retinopathy of prematurity (ROP), were written by Theodore L Terry in the early 1940s, who described several abnormalities in the ocular vasculature of infants born prematurely, the cause of which was unknown. ${ }^{1,2}$ Around this time, clinical studies by Wilson et al found improved respiration in preterm babies when given high concentrations of oxygen, ${ }^{3}$ and so the use of supplemental oxygen therapy for premature neonates became increasingly routine and widespread in the $1950 \mathrm{~s} .{ }^{4}$ Concurrent with this rise in the liberal use of oxygen therapy in the neonatal setting, however, came a well-documented increase in the incidence of ROP. ${ }^{4-7}$ Thaddeus S Szewczyk was one of the first physicians to make the connection between oxygen and ROP from his clinical observations of changes in ROP disease pattern with changes in oxygen concentration administered. ${ }^{8,9}$ However, an intense controversy developed as to how oxygen could cause ROP. This debate could be divided into four general views: anoxia, toxic effects of high oxygen, relative anoxia following high oxygen administration, and factors unrelated to oxygen. ${ }^{10}$ These clinical observations and the ensuing debates sparked research in the early 1950 s to further explore the role of oxygen in ROP using various animal models.
Correspondence: Kip M Connor

243 Charles Street, Boston,

MA 02114, USA

Tel +l 6175736949

Fax +l 6175733078

Email Kip_connor@meei.harvard.edu submit your manuscript | www.dovepress.com

Dovepress

http://dx.doi.org// 0.21 147/EB.S94447 (c) (i) (5) $2016 \mathrm{Kim}$ et al. This work is published and licensed by Dove Medical Press Limited. The full terms of this license are available at https://www.dovepress.com/terms.php BC and incorporate the Creative Commons Attribution - Non Commercial (unported, v3.0) License (http://creativecommons.org/licenses/by-nc/3.0/). By accessing the work you hereby accepe lerms. Non-commercial uses of the work are permitted without any further permission from Dove Medical Press Limited, provided the work is properly attributed. For permission for commercial use of this work, please see paragraphs 4.2 and 5 of our Terms (https://www.dovepress.com/terms.php). 


\section{Initial animal studies of the retinal vasculature}

Decades before the initial oxygen-induced retinopathy (OIR) experiments in the 1950s, researchers were aware that the retina has a relatively higher rate of respiration and anaerobic glycolysis than most other tissues, and that developing retinas exhibit higher oxygen consumption than mature retinas. ${ }^{11}$ Normal retinal vascular development was thoroughly studied in humans, rats, and cats by Michaelson in the 1940s, who found that vascular growth and patterning was overall similar among the different animals. ${ }^{12}$ In light of these earlier findings and clinical observations of ROP, Ashton et al were among the first to explore the effect of changing oxygen concentrations on retinal vascular development in animals, using kittens given their close resemblance to human infants in terms of retinal vascular development. ${ }^{13}$ By varying the onset, duration, and concentration of oxygen exposure in newborn kittens, they were able to characterize two phases of OIR ("vaso-obliterative" and "vaso-proliferative") that closely mimic the early stages of ROP. ${ }^{10}$ Despite the close approximation of this early kitten model of OIR to human ROP, obtaining adequate sample sizes to thoroughly assess a range of experimental variables posed a significant challenge, as noted by the authors at the time..$^{10,14}$

\section{Early murine models of OIR}

The pathologic changes in the mouse retina exposed to hyperoxic conditions that resembled human ROP (eg, hemorrhages, retinal folding, fibrovascular changes in the vitreous body) were first noted around the same time as Ashton's studies in kittens. ${ }^{15,16}$ Given the persistent discussion over the pathophysiology of ROP, many of the early experiments focused heavily on exploring the effects of low oxygen on retinal vascular development. Such experiments helped to disprove the theory that hypoxia was the sole driver of the pathologic retinal neovascularization (NV) seen in ROP and helped to establish hyperoxia as a key inciting factor. ${ }^{10,14,16}$ Subsequent studies in mice reproduced the pathologic ocular findings seen in ROP, albeit to varying degrees and under differing experimental conditions. ${ }^{11,17}$ Although this variability prevented definitive conclusions or extrapolations, these early attempts to generate OIR in mice provided insights that would ultimately prove valuable to the model used today.

Gyllensten and Hellström were the first to document and consistently utilize a specific mouse strain for their OIR experiments in order to minimize the frequency of spontaneous eye anomalies that could complicate their results. ${ }^{14}$ Interestingly, the strain they selected, $\mathrm{C} 57 \mathrm{Bl} / 6$ mice, is one of the most commonly used strains for OIR studies today. Though most preliminary mouse studies involved high oxygen exposure at birth, Gerschman et al studied mice put in high oxygen at different times during their first week of life, ${ }^{11}$ which would prove to be an important distinction given the dramatic changes in the mouse retinal vasculature with each day of development. ${ }^{17}$ Further investigations into mouse retinal vascular development noted an inverse relationship between hyaloid and retinal vessel growth: hyaloid vessels are present at birth but regress over time, while retinal vessels are nearly absent at birth but proliferate almost fully by the first week of life. ${ }^{17}$ This is a particularly critical observation, since early studies noted dilation and proliferation of hyaloid vessels during hyperoxia ${ }^{16-18}$ but were unable to distinguish this hyaloidopathy from true retinopathy, ${ }^{19}$ which may have contributed to the often conflicting results produced by various research groups.

\section{Subsequent advances leading to the current mouse model of OIR}

There was a relative paucity of new work on the mouse model of ROP in the few decades following the 1950s. ${ }^{20}$ Although Ashton's and Patz's animal models of OIR were generally considered to be the most reliable for studying retinal NV, ${ }^{21}$ there were still considerable discrepancies in OIR reproducibility. ${ }^{20}$ For instance, several groups disagreed on the threshold of oxygen exposure that was needed to trigger retinopathy: OIR was observed in kittens with $40 \%$ oxygen, ${ }^{10}$ but in mice, $70 \%$ oxygen was insufficient, ${ }^{22}$ and retinopathy was only observed at $80 \%$ oxygen. ${ }^{16}$ These observations led to the generalization that OIR can only be reliably reproduced at near complete oxygen saturation. ${ }^{20}$ Further compounding the establishment of a standardized mouse OIR model was the lack of a uniform method to assess the severity of retinopathy, which was likely due in part to technological barriers.

Several advancements, from improved retinal vascular imaging to a better understanding of angiogenesis, set the stage for establishing a more rigorous and reliable mouse model of OIR. The advent in 1961 of fluorescein angiography for retinal vascular imaging by Novotny allowed for better visualization of smaller retinal vessels as well as in vivo retinal imaging. ${ }^{23,24}$ Lectin staining, which uses carbohydrate-binding proteins and is the most frequently used technique today for mouse OIR, was first advanced by Blanks and Johnson in the 1980s, who demonstrated that lectins selectively label retinal vasculature in developing mice. ${ }^{25}$ Further studies confirmed the superiority of fluorescein and lectin staining in visualizing retinal vessels during 
mouse development. ${ }^{26}$ Refinements to fluorescein staining, such as labeling dextrans with high-molecular weight fluorescein, minimized contrast leakage and allowed for better visualization of the entire rodent retina at higher resolutions compared to India ink, which was most widely used in the OIR experiments of the 1950s. ${ }^{27}$

In the early 1970 s, Folkman et al reported that ocular NV was triggered by tumor implants in the cornea ${ }^{28}$; this indirectly demonstrated the existence of angiogenic factors and effectively established the field of angiogenesis. This was a particularly significant discovery, since Michaelson and Ashton first postulated the existence of a "vaso-proliferative factor" responsible for the NV seen in ROP decades earlier. ${ }^{10,12,21}$ Another critical finding was the identification ${ }^{29}$ and partial purification ${ }^{30}$ of a retinal substance isolated from different animals that stimulated in vitro endothelial cell growth, which was the first direct evidence of angiogenic activity in the mammalian retina. ${ }^{21}$ This discovery marked a major transition in research, from focusing on speculations and theories of retinal angiogenic activity to identifying specific angiogenic factors and mechanisms. Thus, these pioneering studies ${ }^{30,31}$ inspired further research into retinal angiogenesis, ${ }^{21}$ primarily in mice, given their rapid and proliferative breeding patterns. ${ }^{20}$

Despite the scarcity of new literature on mouse OIR from the late 1950 s to early 1990 s, a few critical refinements in experimental techniques and understanding were made during this time frame. As researchers gradually came to better understand the pathophysiology underlying the two phases of OIR, mouse studies demonstrated that NV was most pronounced after newborn pups were put in hyperoxia for 5 days and then put in normoxia for 5 days. ${ }^{32}$ This resembles the time course of oxygen exposure in the current mouse OIR model that maximizes NV, albeit using an earlier onset of hyperoxia exposure. Researchers had also noted that the extent of vaso-obliteration during phase 1 of OIR was positively correlated with the likelihood of developing NV during phase 2 of OIR. ${ }^{21}$ This observation now serves as the basis for therapeutic strategies that indirectly reduce retinal NV by minimizing early vaso-obliteration. Although there was no consensus regarding an optimal method to assess the severity of retinopathy in mice, ${ }^{20}$ a systematic grading system was established in the kitten model that assessed retinal flatmounts using four categories: extent of vessel growth, vessel pattern, degree of peri-arteriolar capillary free zone, and degree of capillary tuft formation. ${ }^{33}$ Some researchers felt that the small size of the mouse pup retina posed a challenge for successful retinal flatmounts, and that alternative approaches should be pursued, such as manually counting vitreous capillaries in histological cross sections that passed through the optic nerve. ${ }^{20}$ Ultimately, research by Smith et al greatly improved the quantitative assessment of OIR changes by distinguishing hyaloidopathy from retinopathy, ${ }^{19}$ but quantifying NV continues to remain a challenge, as evidenced by the variety of approaches in the past 20 years. ${ }^{34-36}$ The challenges of quantification are explored in further detail later in this review.

\section{Essential concepts of OIR Comparison of human and murine retinal vascular development}

There are several key differences between human and murine retinal developmental angiogenesis that allow the mouse model of OIR to adequately reflect human ROP. One major distinction is the temporal nature of retinal angiogenesis. Whereas development begins relatively early in human fetal development, it occurs postnatally in mice. ${ }^{36,37}$ As a result, human infants born at term possess fully mature retinal vessels, while newborn mice have immature vascular development similar to that seen in premature humans infants. ${ }^{38}$ Fortunately, murine retinal vessel growth progresses in a rapid, predictable, and tightly regulated manner, ${ }^{36,39}$ with near-complete extension of the superficial vascular plexus achieved within a week (with some variability among different mouse strains). ${ }^{40}$ This difference allows for the study and manipulation of retinal vasculature patterning, thereby providing a suitable model for human ROP, which is triggered by subjecting immature retinal vessels to relative hyperoxia at birth.

As mentioned earlier, ROP classically progresses in two phases: initial vaso-obliteration of immature vessels followed by pathological NV during the subsequent vascular repair phase. Granted, advancements in our understanding of ROP pathophysiology have led to the updated notion that phase 1 of $\mathrm{ROP}$ reflects more of a delay in retinal vascular development with modest vaso-obliteration in most cases. ${ }^{37}$ Nevertheless, vaso-obliteration and NV remain the hallmarks of ROP, with the two processes temporally and pathophysiologically linked. The mouse model of OIR consistently reproduces these two key features over the span of 3 weeks with the advantage of genetic manipulation. ${ }^{19,34,36}$ Additionally, the mouse OIR model exhibits spontaneous regression of the new vessels in the late stages of phase 2 , which is consistent with the spontaneous involution seen in many cases of human ROP. ${ }^{41}$ An in-depth comparison of human and murine retinal angiogenesis is provided in the review by Stahl et al. ${ }^{36}$ 


\section{Current mouse model of OIR}

The model developed by Smith et al, ${ }^{19}$ coupled with the method of quantifying vaso-obliteration and pathological $\mathrm{NV}$ established by Connor et al, ${ }^{34}$ is widely regarded as the current standard for OIR experiments in mice. In this model (Figure 1), mouse litters (C57Bl/6 mice are the most widely utilized) are placed in $75 \%$ oxygen at postnatal day (P)7, when there is an optimal balance between hyaloid vessel regression and immature retinal vasculature to allow visualization of subsequent NV. ${ }^{19,32}$ The hyperoxic conditions mimic the relative hyperoxia that premature human infants are exposed to at birth; thus, P7-P12 defines phase 1 of OIR, during which immature vessels regress (Figure 1B) ${ }^{34}$ Regarding the kinetics of phase 1 of OIR, the majority of the vasoobliteration occurs within the first 48 hours of high oxygen exposure. ${ }^{34,42}$ Vessel regrowth has been observed as early as $\mathrm{P9}$, while the mice are still in hyperoxic conditions ${ }^{42,43}$; this may be a response to increasing nutritional demands of the developing retina in the setting of marked vessel loss..$^{36,44}$ The transfer of mice to room air at P12 results in hypoxia in the regions of vaso-obliteration that stimulates pro-angiogenic factors. ${ }^{36}$ This is discussed in further detail in the next section. Vessel growth induced by these angiogenic factors includes regrowth of normal vessels into areas of vaso-obliteration and pathological NV at the junction of avascular and vascularized retina (Figure 2). These processes occur simultaneously but to varying degrees depending on factors such as time course ${ }^{45}$ and extent of vessel regression during phase $1^{21,36}$; however, the mechanisms that govern normal versus pathological vessel growth are still poorly understood. ${ }^{45}$

There has been some variability in how NV is assessed and quantified, as we explore later in this review, but retinal $\mathrm{NV}$ is generally defined as an unorganized configuration of leaky, small-caliber vessels (ie, preretinal tufts) located anterior to normal retinal vessels with frequent extension into the vitreal space (Figure 1C). ${ }^{21,36} \mathrm{NV}$, which develops beginning at $\mathrm{P} 12$ with rapid proliferation starting at $\mathrm{P} 14$ and peak density at P17, ${ }^{19,42}$ is the hallmark of phase 2 of OIR and the primary factor responsible for vision impairment. As vascular repair continues and levels of pro-angiogenic factors wane, NV begins to regress after P17 with almost complete resolution and replacement by normal vasculature by P25 (Figure 1D), ${ }^{36}$ reflecting an inverse trend between normal revascularization and $\mathrm{NV}^{45}$ Although retinas can be harvested for analysis at any point during OIR, studies of phase 1 generally assess vaso-obliteration at P8 or P12, while NV is typically examined at $\mathrm{P} 17 .{ }^{36} \mathrm{~A}$ comprehensive protocol by Connor et al provides detailed instructions on experimental setup, sample collection, data analysis, and troubleshooting. ${ }^{34}$

\section{Role of angiogenic factors}

Through various animal models of NV, researchers have uncovered a vast array of molecular pathways that govern developmental and pathological retinal angiogenesis. Among these, the role of vascular endothelial growth factor (VEGF) has been the most extensively studied, and VEGF is considered one of the most important mediators in this context. Initially identified as a modulator of vessel permeability, ${ }^{46,47}$ VEGF is a family of polypeptides produced as multiple isoforms that act via tyrosine kinase receptors. ${ }^{48} \mathrm{As}$ researchers began to explore the functions of VEGF in the eye in the 1990s, it became increasingly evident that VEGF was likely the elusive "vaso-proliferative factor" initially posited by Michaelson and Ashton almost half a century earlier. ${ }^{48}$ The role of VEGF in ocular angiogenesis was initially demonstrated using a model of photocoagulation-induced ischemia in nonhuman primates. These studies revealed that ischemia-induced VEGF production in the retina is clearly associated with increased ocular NV and vascular permeability in vivo. ${ }^{49,50}$ Expanding on this work, Pierce et al explored the role of VEGF using the mouse OIR model, further strengthening the notion that VEGF induces pathologic retinal NV. ${ }^{51}$ Stone et al demonstrated that VEGF expression is essential in normal retinal vascular development, and that high oxygen levels suppress VEGF production, thus inhibiting vessel growth and leading to the regression of nascent immature vessels. ${ }^{52}$ Subsequently, Aiello et al showed that inhibition of VEGF suppresses murine retinal NV in vivo. ${ }^{53}$ Taken together, these findings formed the basis for the development of anti-VEGF therapies that are now the mainstay for the treatment of choroidal NV in wet age-related macular degeneration. ${ }^{36}$

Within the OIR model, retinal VEGF expression predictably correlates with disease progression. High oxygen exposure during phase 1 suppresses retinal VEGF levels and results in central retinal vaso-obliteration. ${ }^{36,54,55}$ Upon return to room air, hypoxia in the central retina leads to the upregulation of VEGF, primarily by retinal glial cells, which in turn leads to the NV that is characteristic of phase $2 \cdot{ }^{36,51,54}$ The mechanisms that distinguish pathologic from developmental retinal angiogenesis are not fully understood, although it is clear that VEGF activity is vital to both processes. ${ }^{52,54}$ Independent of VEGF activity, the alternative complement pathway, a key component of innate immunity, has recently been shown to aid in the clearance of pathologic NV without damaging 
A
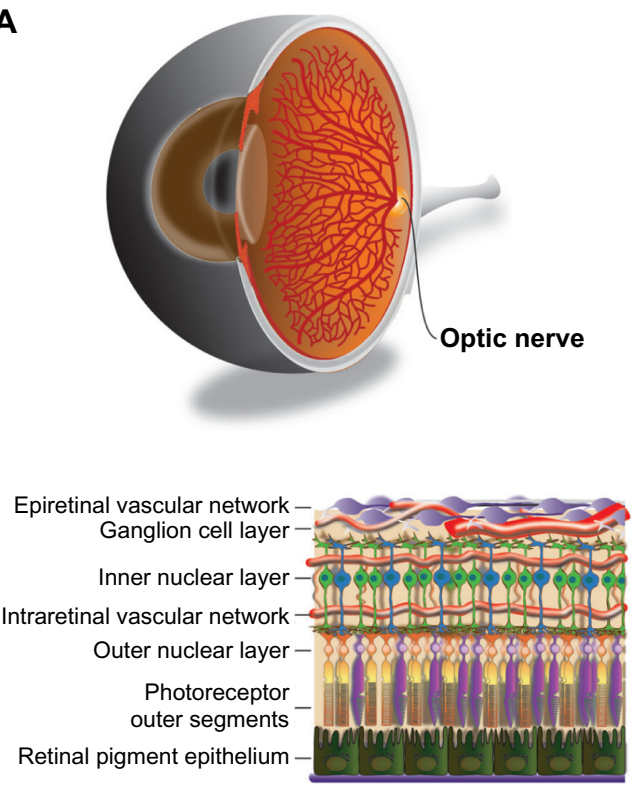

C

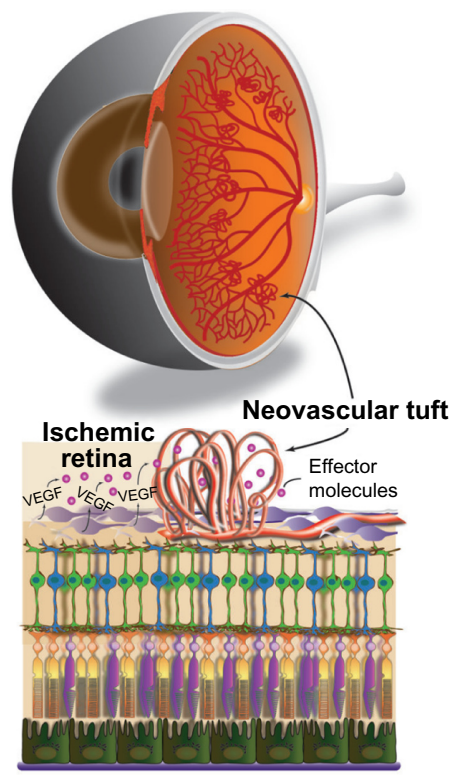

B

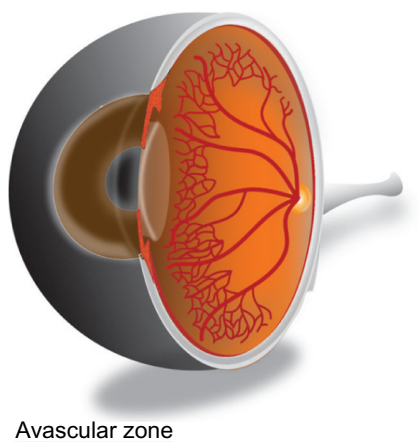

Avascular zone
Relative hyperoxia

IVEGLVE, LVEGF

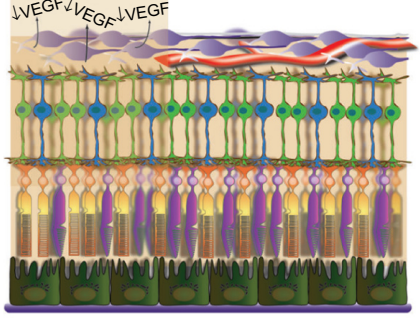

D

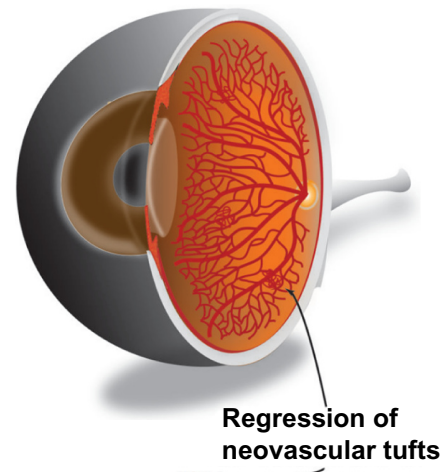

anto

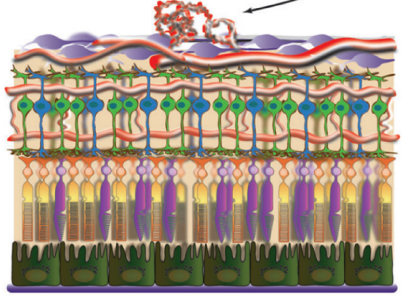

Figure I Murine retinal vascular changes during OIR.

Notes: (A) Graphic representation of retinal vasculature at the microscopic level (superior image) and in microscopic cross section (inferior image) in a healthy C57BI/6 mouse at P7 under normal conditions. Mice are normally born with avascular retinas, allowing for full observation of vascular growth postnatally. By P7, the superficial vascular plexus (ie, epiretinal vascular network) almost fully extends across the peripheral retina, and vessels begin to develop orthogonal to the retinal surface, eventually forming the intermediate and deep vascular plexus (ie, intraretinal vascular network). Full vascular maturation is usually achieved around P2I-P25 in C57BI/6 mice. (B) Depiction of vaso-obliteration during phase I of OIR, when mice are exposed to $75 \%$ oxygen from P7 to PI2. An important distinction is that vaso-obliteration in mice occurs in the central retina, in contrast to the peripheral vaso-obliteration seen in the rat OIR model and in human ROP. During this high oxygen exposure, VEGF production is suppressed in the relatively hyperoxic central retina, resulting in vascular loss. Of note, progression of vaso-obliteration is most rapid within the first 48 hours of high oxygen exposure. (C) Upon transfer from high oxygen exposure to room air at PI2, the central avascular retina experiences relative ischemia, especially given the nutritional demands of the growing retina. As a result, VEGF is produced in this ischemic zone, which activates downstream effector molecules that lead to the characteristic neovascular tufts seen in phase 2 of OIR. Neovessels primarily form at the junction of the vascular and avascular zones, with peak neovessel density reached by PI7 in C57BI/6 mice, as depicted here. (D) Depiction of the vascular repair process, where spontaneous neovessel regression coincides with waning pro-angiogenic factors and normal revascularization. This repair process is essentially complete by P25.

Abbreviations: OIR, oxygen-induced retinopathy; P, postnatal day; ROP, retinopathy of prematurity; VEGF, vascular endothelial growth factor.

normal retinal vasculature. ${ }^{56}$ Normally, retinal vessels express inhibitors of the complement system that prevent their clearance. However, a downregulation of complement inhibitors was observed exclusively on pathologic neovessels during phase 2 of OIR, thus allowing for their targeted removal by the alternative complement pathway. ${ }^{56}$ Another important consideration is that specific VEGF isoforms have different roles in angiogenesis ${ }^{57}$; murine VEGF164, which is 


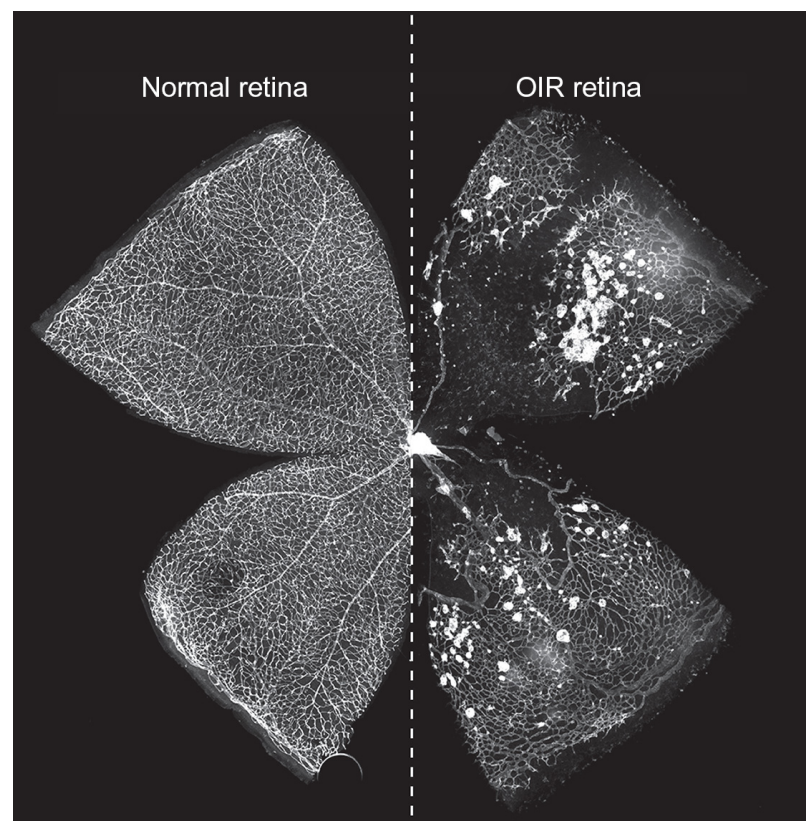

Figure 2 Control and OIR flatmounts at murine PI7.

Notes: In this composite image, retinal flatmounts from C57BI/6 mice at PI7 under normal (left) and OIR (right) conditions were stained with isolectin B4 to label the vasculature (white). Normally, as seen on the left, retinal vessels branch radially from the optic disk in a uniform pattern across the entire retina. In contrast, PI7 OIR mouse retinas possess extensive central vaso-obliteration with pathologic neovessels forming around the junction of the vascular and avascular zones, as evidenced by the dense, circular lectin-stained clusters seen on the right. These leaky, unorganized tufts often extend into the vitreous and reach peak density at $\mathrm{PI7}$, which is when neovascularization is typically assessed. Abbreviations: OIR, oxygen-induced retinopathy; $\mathrm{P}$, postnatal day.

analogous to human VEGF165, is the predominant isoform in the retina. ${ }^{53,56} \mathrm{In}$ addition, different angiogenic responses have been observed in OIR models depending on the type of VEGF receptor activated ${ }^{36}$; it is thought that VEGF receptor-1 is involved in vascular permeability and chemotaxis, while VEGF receptor-2 is important for the survival and proliferation of endothelial cells. ${ }^{58}$ Finally, it is important to note that VEGF is just one of many angiogenic factors that have been implicated in OIR; other factors, including erythropoietin ${ }^{59}$ and insulin-like growth factor 1 (IGF-1, explored later in this review), ${ }^{60}$ and omega-3-polyunsaturated fatty acids ${ }^{61}$ play notable roles as well.

\section{Limitations of the mouse model of OIR}

\section{Strain and litter differences in OIR phenotype}

Despite the improved reproducibility and widespread adoption of the mouse model of OIR, several important factors can result in considerable variability in the OIR phenotype if not addressed. Among these, strain-dependent variability has been a longstanding issue. Indeed, strain-dependent differences have plagued the use of the rat OIR model and may have been an obstacle to the widespread adoption of the model. ${ }^{62}$ Many of the mouse OIR studies in the 1950s did not specify the strain type, and we now know that this partially contributed to the divergent results among research groups. Gyllensten and Hellström were among the first to explicitly emphasize the importance of the consistent use of a single mouse strain, $\mathrm{C} 57 \mathrm{Bl} / 6$, given its low frequency of spontaneous eye abnormalities. ${ }^{14}$ Through advances in our understanding of angiogenesis and progress in genetic techniques, it has been shown that there is considerable genetic heterogeneity in ocular angiogenesis in different strains of mice, both in response to exogenous angiogenic factors ${ }^{63}$ and in the resting vasculature. ${ }^{64}$ In terms of OIR, straindependent differences have been observed in the context of light exposure ${ }^{65}$ and expression of angiogenic factors. ${ }^{66}$ Interestingly, C57Bl/6 mice were found to have increased photoreceptor vulnerability to oxygen stress compared to the BALB/cJ mouse strain, ${ }^{65}$ and lower levels of angiogenesis compared to $129 \mathrm{~S} 3 /$ SyIM mice. ${ }^{66}$ Even when accounting for strain-dependent differences in OIR susceptibility, variability is still observed in strains purchased from different vendors, likely attributable to spontaneous mutations that have accumulated from generational inbreeding. ${ }^{36}$ It is also important to account for variance within a given mouse litter, ${ }^{34,36,67}$ which we explore in further depth through our discussions on postnatal weight gain (PWG) and hyperoxiadependent changes on nursing mothers. In order to address these potential confounders, mouse OIR experiments should be conducted using the same strain obtained from a single vendor, intra-litter controls should be included when possible to minimize age and genetic heterogeneity, and there should be equal sampling from multiple litters. These variables are particularly important for studies using transgenic mice, in which the experimental and control mice should be from the same genetic background..$^{34,36}$

\section{Effect of hyperoxia on lactating mothers}

One notable challenge with the use of the mouse OIR model is the toxicity of hyperoxia, not only for pups but also for their nursing mothers. The earliest OIR experiments reported that nursing mothers were less tolerant of hyperoxic conditions than their pups, prompting the use of surrogate mothers from room air controls. ${ }^{11}$ Surrogate use in the mouse OIR model has varied among research groups but was often preemptively incorporated in anticipation of known complications, such as pulmonary oxygen toxicity. ${ }^{20}$ However, the currently accepted OIR model limits room air surrogate use to only when the 
mother expires, a relatively rare occurrence ${ }^{19}$ that generally occurs on the last day of high oxygen exposure or upon return to room air (ie, P12). ${ }^{34}$ Nevertheless, the stress of hyperoxia on nursing mothers can be potentially detrimental to nursing behavior and fertility. It has been reported that the detrimental effects of hyperoxia to the mother can be minimized by modifying the model to 22 hours of high oxygen and 2 hours of room air per day instead of continuous high oxygen exposure ${ }^{68}$ From our laboratory's experience, nursing mothers are no longer fertile after exposure to OIR, and thus should not be used for breeding purposes. We have also observed an increased tendency for mothers to cannibalize their pups during exposure to hyperoxia, as well as after transfer to normoxia when their cages are handled frequently. In contrast to the OIR studies of the 1950s when pups were routinely and frequently taken from the chambers for examination and returned, ${ }^{11}$ we recommend minimal handling after return to normoxia as the stress from handling can lead to decreased lactation and increased cannibalization. Since mouse pups are dependent on their nursing dam for nutrition for the first 21 days of life, essentially the time course of OIR, the health and behavior of the nursing mother or surrogate are critical factors in pup survival. We have found that switching nursing mothers with surrogates from the 129S mouse strain upon transfer to room air at P12 greatly improves PWG and survival of pups. In spite of this, it is not uncommon for OIR pups to expire more frequently than room air pups for a variety of reasons, ranging from the aforementioned maternal factors to pup nutritional status, which we explore next in this review. Thus, when calculating sample sizes, it is important to anticipate the poor survival of pups exposed to OIR conditions.

\section{Postnatal weight gain and OIR}

Despite decades of experience with the mouse OIR model, PWG has only recently come to the attention of researchers as a critical factor in disease progression. The topic has been of increasing interest in clinical studies of ROP in recent years, especially since clinical advances that account for supplemental oxygen level with respect to gestational age have decreased ROP incidence and severity. ${ }^{69,70}$ Indeed, screening methods that monitor PWG and IGF-1, a key factor in nutritional intake and retinal angiogenesis, ${ }^{60,71,72}$ have been developed to reliably predict ROP. ${ }^{73-75}$ Nevertheless, the physiological mechanisms behind weight-dependent OIR changes are not entirely known, and this has prompted a more thorough examination of weight gain in mouse models. ${ }^{67,70}$ Prior research in a rat OIR model had noted an inverse relationship between the severity of $\mathrm{NV}$ and final weight. ${ }^{76}$ Vanhaesebrouck et al observed a similar trend in mice; mice from larger litters had lower body weight, lower circulating levels of IGF-1, and more severe OIR.$^{67}$ Furthermore, they found that exogenous administration of IGF-1 was protective against OIR, providing the first strong evidence for IGF-1 as a therapeutic target. These findings highlight the importance of litter size as a proxy for postnatal growth and nutritional status, and current OIR protocols recommend limiting litter size to eight pups. ${ }^{34}$ In another mouse OIR study by Stahl et al, pups with poor PWG (defined as $<5 \mathrm{~g}$ at P17) had delayed and markedly prolonged vaso-obliteration and $\mathrm{NV}$ compared to medium weight gain (defined as 5-7.5 g) and extensive weight gain (defined as $>7.5 \mathrm{~g}$ ) pups. ${ }^{70}$ The effect of surrogate use and litter size was also explored, with the results confirming the notion that smaller litter size (3-4/litter) correlates with improved weight gain and nutritional status, and that the use of additional surrogate dams at P1 leads to improved weight gain and NV similar to that observed in medium weight gain and extensive weight gain pups. ${ }^{70}$ Thus, it is critical to document the use of surrogates in OIR experiments and to be mindful of litter size as these factors can markedly influence PWG and disease course.

In terms of the physiological mechanisms that may drive such changes, expression of VEGF, a potent stimulator of retinal angiogenesis, ${ }^{51,53,55}$ was predictably elevated and prolonged in PWG pups, in a manner consistent with OIR disease course. ${ }^{70}$ Additionally, IGF-1 was reduced early during phase 2 of OIR, which could lead to impaired revascularization. ${ }^{70}$ Taken together, these findings highlight the importance of PWG as an independent factor in OIR disease course and severity. Recommendations have been made to record weight data in all OIR experiments, to exclude mice $<6 \mathrm{~g}$ at $\mathrm{P} 17,{ }^{34}$ and to ensure the use of consistent mouse weights when making comparisons. ${ }^{70}$

\section{Light exposure and OIR}

The potential role of light exposure in ROP pathogenesis was first posited in 1942 by Terry. ${ }^{1}$ Since then, clinical studies on the effect of light exposure on ROP incidence and severity have yielded conflicting results, ${ }^{77,78}$ likely due to the multiplicity of variables in premature infant health and treatment conditions. ${ }^{79}$ Thus, in the 1990s, researchers began to explore this question in animal OIR models, initially using rats ${ }^{80}$ and kittens. ${ }^{81}$ No significant differences in pattern or course of OIR were observed with changes in light exposure duration or intensity in either of these animal models. ${ }^{80,81}$ Expanding these studies to the mouse model of OIR, Wesolowski and 
Smith also did not note any adverse effects of light exposure on OIR but instead found that mice exposed to phase 1 hyperoxia in the dark had more NV than corresponding mice in the light; they hypothesize that this difference may be due to higher metabolic demands of the retina at night. ${ }^{79}$

It is important to note that rodent pups are born with fused eyelids, which means that higher light intensities are needed to reach the eye. ${ }^{82}$ While no light-dependent vascular changes were noted in the OIR rat model, structural photoreceptor damage leading to progressive retinal degeneration has been observed with high-intensity light, ${ }^{80}$ especially in albino Sprague Dawley rats. ${ }^{82}$ Of particular interest, a recent study by Natoli et al demonstrates that exposure to $670 \mathrm{~nm}$ red light during OIR in mouse and rats can protect against ROP, as evidenced by decreased vaso-obliteration, $\mathrm{NV}$, and abnormal vascular patterning. ${ }^{83}$ This novel finding suggests that light exposure may play a more substantial role in OIR and ROP than previously thought. In the context of OIR, time-dependent variability in retinal vascular growth and the changes associated with circadian rhythms may play a bigger role than light exposure, and so it is important to start experiments and sample collection at the same time of day to minimize potential variability from such factors. ${ }^{84}$

\section{Quantifying OIR}

Although the advent of lectin and fluorescein staining greatly improved the visualization of vessels in the mouse retina, assessing the pathologic vascular changes seen in OIR continues to pose a significant challenge. As mentioned previously, a major benefit of Smith's model has been the establishment of a method to quantify NV. ${ }^{19}$ Unlike prior methods that did not distinguish hyaloid and retinal NV, Smith et al were able to more accurately assess retinal NV by manually counting in retinal cross sections the nuclei of the cells of the microvessels that were invading into the vitreous. ${ }^{19}$ While this cross-sectional technique has been utilized in other studies, ${ }^{51,53,67,85}$ the ability to successfully flatmount the whole mouse retina allowed for quantitative NV assessment that was previously thought to be too difficult to accomplish in mice. ${ }^{20}$ The use of retinal whole-mounts has led to the development of a grading system for NV based on predefined characteristics. ${ }^{86,87}$ Since whole retinal flatmount analysis allows the investigator to obtain a full view of the extent of disease (versus a snapshot of particular areas seen in retinal cross sections), counting vascular nuclei in cross sections is no longer regarded as the ideal means to quantify OIR. With the advent of computer-aided imaging software, it became possible to more precisely measure the area of neovascular tufts in mouse retinal whole-mounts. ${ }^{59,61,88,89}$ Furthermore, image tracing and pixel calculations through software like Adobe Photoshop also allowed for the quantification of vaso-obliteration, ${ }^{34}$ thereby providing a more comprehensive assessment of vascular changes throughout both phases of OIR.

Despite the improved accuracy of NV quantification afforded by these updated methods, all protocols are timeintensive and are somewhat subjective, given the need for manual counting, grading, or tracing of $\mathrm{NV}^{35} \mathrm{~A}$ new computer-aided method was developed in 2009 by Stahl et al using the free ImageJ software provided by the National Institutes of Health that makes the quantification process not only about five times faster but also less user-dependent and consequently more objective. ${ }^{35}$ This method, termed SWIFT_NV, employs a set of macros that digitally subtract background fluorescence from overlaid images of retinal flatmounts, thus isolating neovascular tufts from normal vessels. While the approach requires manual tracing of areas of vaso-obliteration in order to overlay images, the authors argue that manual tracing of vaso-obliterated areas is much less time-consuming and subjective than manually tracing $\mathrm{NV}^{35}$ Notably, computer-aided image analysis of retinal NV was utilized in the rat model of OIR ${ }^{90}$ more than a decade prior to its use in the mouse OIR model. Similar to the background subtraction method used in the SWIFT_NV approach that makes quantification more user-independent, Penn and Gay used image overlay and signal intensity threshold algorithms to the same effect $\sim 20$ years earlier. ${ }^{90}$

Even with the improved reliability, objectivity, and efficiency of the SWIFT_NV approach, a few issues still remain. The quantification of vaso-obliterated areas still requires manual tracing, making studies of phase 1 of OIR vulnerable to inter-user variability. Plus, the SWIFT_NV approach itself is technically vulnerable since it requires manual tracing. A possible solution to this potential source of bias would be to incorporate masking into image analysis, where the user would not know which experimental group he or she is quantifying until after analysis is complete. The vaso-obliterative phase of OIR is relatively understudied; however, this phase is likely to receive more attention as it has become well established that the severity of vessel regression in phase 1 influences the extent of later NV. ${ }^{36}$ In addition to quantifying vascular changes in early OIR, another challenge is assessing the resolution of NV during the end stages of OIR. This is inherently difficult, given our relative lack of understanding regarding how pathological vessels regress, ${ }^{34}$ although recent research from our laboratory has implicated 
the alternative complement pathway, a key component of our innate system, in the clearance of pathological NV. ${ }^{56}$ That being said, there is a systematic approach that assesses the resolution of $\mathrm{NV}$ based on four features of retinal vessels: shape, diameter, fluorescent intensity, and extension from the superficial retinal layer. ${ }^{34,91}$

Finally, a major limitation of all of these quantitative approaches is that the mice must be sacrificed to allow for retinal isolation and staining, and so methods for in vivo quantification of NV are needed. Topical endoscopy fundus imaging (TEFI), a technique developed by Paques et al that provides high-resolution digital photographs of the retina in live mice, ${ }^{92}$ has been recently adapted for use in the mouse OIR model. ${ }^{93}$ Although the earliest time point that TEFI can detect retinal vascular changes is at P15, the images obtained reflect disease course that is consistent with conventional methods of assessment, and a 5-point grading system developed by Furtado et al demonstrates reasonable intra- and interobserver reliability. ${ }^{93}$ The use of TEFI provides the opportunity to perform multiple retinal examinations in the same mouse to track disease course. ${ }^{93}$ Another recent technique, proposed by Mezu-Ndubuisi et al, couples fluorescein angiography with oxygen-sensitive microelectrodes to quantify NV changes during OIR in live mice. ${ }^{94}$ Although neither technique is as accurate as conventional methods, they allow each sample to serve as its own control, thus strengthening the robustness of OIR data.

\section{Additional considerations}

There are a few other issues to consider when using the mouse model of OIR. Genetic or immunohistochemical studies that require RNA or protein, respectively, may be hindered by the smallness of neonatal mouse pup retinas. Thus, retinas from a number of mice are often pooled to obtain adequate protein or RNA levels for quantification. Ishikawa et al have recommended that both eyes from the same mouse pup be used for RNA studies, four eyes from two pups raised under identical conditions be used for enzyme-linked immunosorbent assay, and biological replicates be used to reduce variability. ${ }^{95}$ For gene expression studies, it is important to keep in mind that variable oxygen tensions can induce differential gene expression within different regions of the mouse retina. ${ }^{96}$

Sex-related differences in OIR have not been thoroughly explored, possibly because of the difficulty in determining the sex of mouse pups. Smith et al claimed no such differences in their original 1994 OIR study, but they had also claimed no weight-related OIR differences in the same study, ${ }^{19}$ which we know not to be true. Interestingly, Miyamoto et al have found that administration of estrogen, specifically the ovarian sex hormone E2, during either phase of OIR can modulate VEGF expression and mitigate OIR severity. ${ }^{97}$ In humans, it has been observed that female preterm infants with very low birth weights have a slight survival advantage compared to their male counterparts. ${ }^{98,99}$ Although clinical studies have not observed sex-related differences in ROP incidence, ${ }^{100}$ Yang et al found that sex was an independent risk factor for ROP that warrants surgery, with male infants more likely to undergo such procedures. ${ }^{101}$ Regardless of whether the sex of mouse pups affects OIR disease course, modulation of sex hormones may play a therapeutic role for ROP in the clinical setting.

Finally, it is worth noting that the mouse model of OIR does not replicate certain aspects of ROP seen in humans. Given improvements in supplemental oxygen dosing for preterm infants, the constant exposure of mice to $75 \%$ oxygen is much higher and less cyclical than what premature human infants experience, ${ }^{37}$ which is actually closer to the $50 / 10$ fluctuating OIR model in rats. ${ }^{38}$ Additionally, no gestational manipulation occurs with the mouse OIR model since experiments are conducted postnatally, which means that other complications of premature birth are not reproduced. ${ }^{37}$ While other animal OIR models (eg, kittens, beagles, rats) more closely resemble human ROP, ${ }^{37,102}$ the mouse OIR model remains the quickest and least expensive, with the broadest transgenic and antibody-staining capabilities.

\section{Conclusion}

\section{Application of the mouse OIR model to current and future research}

A Google Scholar search of "oxygen induced retinopathy mice" (July 29, 2015) indicates that there are $\sim 35,500$ publications to date on the mouse model of OIR, with about half of these publications coming after the last such search conducted by Stahl et al in 2009. ${ }^{36}$ This vast number reflects the ease with which researchers using this model can investigate a broad range of topics. Among these, the role of the immune system has been of particular interest, with hypotheses on the role of inflammation in ocular NV well preceding Smith's work. ${ }^{103}$ The complement system, a fundamental component of innate immunity, has recently been studied in the context of OIR, and has been shown to mediate retinal angiogenesis through clearance of pathologic neovessels. ${ }^{56,104}$ Although leukocytes have also been shown to mediate retinal vascular remodeling in OIR, ${ }^{105}$ neuroinflammation in the retina is still a relatively unexplored topic. Potential neurovascular crosstalk, specifically between retinal glial and neuronal cells, is becoming of interest in this context. ${ }^{106-108}$ Additionally, the 
role of oxidative stress has been aptly studied in relation to the model, ${ }^{109-112}$ with recent work demonstrating a critical role for endogenous and synthetic antioxidants in countering OIR. ${ }^{68,113}$ Modulation of beta-adrenergic receptor activity has been shown to alter angiogenic activity in OIR. ${ }^{14,115}$ Indeed, insights gained from the mouse OIR model, coupled with technological advancements in immunostaining and genetic manipulation, may help improve or lead to new animal models of vaso-proliferative retinopathies, ${ }^{116}$ such as the development of a zebrafish model of hypoxia-induced retinopathy. ${ }^{117,118}$ Undoubtedly, the impact of the mouse OIR model has been significant: what began as an attempt to replicate ROP in mice has developed into a robust platform for investigating angiogenesis, neuroinflammation, and novel therapeutic strategies for many ocular conditions.

\section{Acknowledgments}

This study was supported by US National Institutes of Health/National Eye Institute Grant R01EY022084/S1 (to KMC), Research to Prevent Blindness (RPB) Special Research Scholar Award (to KMC), RPB Medical Student Fellowship Grant (to CBK), and a Fight for Sight, Inc. Student Fellowship Award (to CBK).

\section{Disclosure}

The authors report no conflicts of interest related to this work.

\section{References}

1. Terry TL. Fibroblastic overgrowth of persistent tunica vasculosa lentis in infants born prematurely: II. Report of cases-clinical aspects. Trans Am Ophthalmol Soc. 1942;40:262-284.

2. Terry TL. Retrolental fibroplasia in the premature infant: V. Further studies on fibroplastic overgrowth of the persistent tunica vasculosa lentis. Trans Am Ophthalmol Soc. 1944;42:383-396.

3. Wilson JL, Long SB, Howard PJ. Respiration of premature infants. Am J Dis Child. 1942;63(6):1080.

4. Tin W, Gupta S. Optimum oxygen therapy in preterm babies. Arch Dis Child Fetal Neonatal Ed. 2007;92(2):F143-F147.

5. Bedrossian RH, Carmichael P, Ritter J. Retinopathy of prematurity (retrolental fibroplasia) and oxygen. Am J Ophthalmol. 1954;37(1):78-86.

6. Zacharias L, Reynolds WE, Chisholm JF, King MJ. The incidence and severity of retrolental fibroplasia in relation to possible causative factors. I. Observations on the occurrence of retrolental fibroplasia. II. Studies of the relationship of retrolental fibroplasia to degree of prematurity, oxygen therapy, general health, and date of birth of premature infants. Am J Ophthalmol. 1954;38(3):317-336.

7. Parmelee AH, Pilger IS, Austin WO. Retrolental fibroplasia; a reduction in incidence following a decrease in use of oxygen therapy for premature infants. Calif Med. 1956;84(6):424-426.

8. Szewczyk TS. Retrolental fibroplasia; etiology and prophylaxis. Am J Ophthalmol. 1952;35(3):301-311.

9. Szewczyk TS. Retrolental fibroplasia and related ocular diseases; classification, etiology, and prophylaxis. Am J Ophthalmol. 1953;36(10): 1336-1361.

10. Ashton N, Ward B, Serpell G. Effect of oxygen on developing retinal vessels with particular reference to the problem of retrolental fibroplasia. Br J Ophthalmol. 1954;38(7):397-432.
11. Gerschman R, Nadig PW, Snell AC, Nye SW. Effect of high oxygen concentrations on eyes of newborn mice. Am J Physiol. 1954;179(1): $115-118$.

12. Michaelson IC. The mode of development of the vascular system of the retina, with some observations on its significance for certain retinal diseases. Trans Ophthalmol Vis Sci. 1948;68:137-180.

13. Ashton N, Ward B, Serpell G. Role of oxygen in the genesis of retrolental fibroplasia. Br J Ophthalmol. 1953;37(9):513-520.

14. Gyllensten LJ, Hellström BE. Experimental approach to the pathogenesis of retrolental fibroplasia. Br J Ophthalmol. 1955;39(7):409-415.

15. Gyllensten LJ, Hellström BE. Retrolental fibroplasia; animal experiments: the effect of intermittently administered oxygen on the postnatal development of the eyes of full term mice. Acta Paediatr. 1952;41(6):577-582.

16. Patz A, Eastham A, Higginbotham DH, Kleh T. Oxygen studies in retrolental fibroplasia. Am J Ophthalmol. 1953;36(11):1511-1522.

17. Habegger H, Randolf Philbrook F, Curley FJ, Ingalls TH. Retinopathy of immaturity. Am J Ophthalmol. 1956;42(3):377-392.

18. Gyllensten LJ, Hellström BE. Experimental approach to the pathogenesis of retrolental fibroplasia. I. Changes of the eye induced by exposure of newborn mice to concentrated oxygen. Acta Paediatr Suppl. 1954;43(100):131-148.

19. Smith LEH, Wesolowski E, McLellan A, et al. Oxygen-induced retinopathy in the mouse. Invest Ophthalmol Vis Sci. 1994;35(1):101-111.

20. Gole GA, Browning J, Elts SM. The mouse model of oxygen-induced retinopathy: a suitable animal model for angiogenesis research. Doc Ophthalmol. 1990;74(3):163-169.

21. Patz A. I. Studies on retinal NV. Invest Ophthalmol Vis Sci. 1980;10(10): $1133-1138$.

22. Bischoff PM, Wajer SD, Flower RW. Scanning electron microscopic studies of the hyaloid vascular system in newborn mice exposed to $\mathrm{O}_{2}$ and $\mathrm{CO}_{2}$. Graefe's Arch Clin Exp Ophthalmol. 1983;220(6): 257-263.

23. Novotny HR, Alvis DL. A method of photographing fluorescence in circulating blood in the human retina. Circulation. 1961;24:82-86.

24. Keane PA, Sadda SR. Retinal imaging in the twenty-first century. Ophthalmology. 2014;121(12):2489-2500.

25. Blanks JC, Johnson LV. Selective lectin binding of the developing mouse retina. J Comp Neurol. 1983;221(1):31-41.

26. Connolly SE, Hores TA, Smith LEH, D'Amore PA. Characterization of vascular development in the mouse retina. Microvasc Res. 1988;36(3):275-290.

27. D'Amato R, Wesolowski E, Smith LE. Microscopic visualization of the retina by angiography with high-molecular-weight fluorescein-labeled dextrans in the mouse. Microvasc Res. 1993;46(2):135-142.

28. Folkman J, Merler E, Abernathy C, Williams G. Isolation of a tumor factor responsible for angiogenesis. J Exp Med. 1971;133(2):275-288.

29. Glaser BM, D'Amore PA, Michels RG, et al. The demonstration of angiogenic activity from ocular tissues. Preliminary report. Ophthalmology. 1980;87(5):440-446.

30. Glaser BM, D'Amore PA, Michels RG, Patz A, Fenselau A. Demonstration of vasoproliferative activity from mammalian retina. J Cell Biol. 1980;84(2):298-304.

31. Folkman J, Klagsbrun M. Angiogenic factors. Science. 1987;235(4787): 442-447.

32. Ashton N. Some aspects of the comparative pathology of oxygen toxicity in the retina. Ophthalmologica. 1970;160(1):54-71.

33. Phelps DL, Rosenbaum AL. Effects of marginal hypoxemia on recovery from oxygen-induced retinopathy in the kitten model. Pediatrics. 1984;73(1):1-6

34. Connor KM, Krah NM, Dennison RJ, et al. Quantification of oxygeninduced retinopathy in the mouse: a model of vessel loss, vessel regrowth and pathological angiogenesis. Nat Protoc. 2009;4(11):1565-1573.

35. Stahl A, Connor KM, Sapieha P, et al. Computer-aided quantification of retinal NV. Angiogenesis. 2009;12(3):297-301.

36. Stahl A, Connor KM, Sapieha P, et al. The mouse retina as an angiogenesis model. Invest Ophthalmol Vis Sci. 2010;51(6):2813-2826. 
37. Hartnett ME. Pathophysiology and mechanisms of severe retinopathy of prematurity. Ophthalmology. 2015;122(1):200-210.

38. Madan A, Penn JS. Animal models of oxygen-induced retinopathy. Front Biosci. 2003;8(12):1030-1043.

39. Dorrell MI, Friedlander M. Mechanisms of endothelial cell guidance and vascular patterning in the developing mouse retina. Prog Retin Eye Res. 2006;25(3):277-295.

40. Aguilar E, Dorrell MI, Friedlander D, et al. Chapter 6. Ocular models of angiogenesis. Methods Enzymol. 2008;444:115-158.

41. Repka MX, Palmer EA, Tung B. Involution of retinopathy of prematurity Cryotherapy for Retinopathy of Prematurity Cooperative Group. Arch Ophthalmol. 2000;118(5):645-649.

42. Lange C, Ehlken C, Stahl A, Martin G, Hansen L, Agostini HT. Kinetics of retinal vaso-obliteration and $\mathrm{NV}$ in the oxygen-induced retinopathy (OIR) mouse model. Graefe's Arch Clin Exp Ophthalmol. 2009;247(9):1205-1211

43. Gu X, Samuel S, El-Shabrawey M, et al. Effects of sustained hyperoxia on revascularization in experimental retinopathy of prematurity. Invest Ophthalmol Vis Sci. 2002;43(2):496-502.

44. Ozaki H, Yu AY, Della N, et al. Hypoxia inducible factor-1alpha is increased in ischemic retina: temporal and spatial correlation with VEGF expression. Invest Ophthalmol Vis Sci. 1999;40(1):182-189.

45. Scott A, Fruttiger M. Oxygen-induced retinopathy: a model for vascular pathology in the retina. Eye (Lond). 2010;24(3):416-421.

46. Senger D, Galli S, Dvorak A, Perruzzi C, Harvey V, Dvorak H. Tumor cells secrete a vascular permeability factor that promotes accumulation of ascites fluid. Science. 1983;219(4587):983-985.

47. Ferrara N, Henzel WJ. Pituitary follicular cells secrete a novel heparinbinding growth factor specific for vascular endothelial cells. Biochem Biophys Res Commun. 1989;161(2):851-858.

48. D'Amore PA. Mechanisms of retinal and choroidal NV. Invest Ophthalmol Vis Sci. 1994;35(12):3974-3979.

49. Miller JW, Adamis AP, Shima DT, et al. Vascular endothelial growth factor/vascular permeability factor is temporally and spatially correlated with ocular angiogenesis in a primate model. Am J Pathol. 1994;145(3):574-584.

50. Shima DT, Gougos A, Miller JW, et al. Cloning and mRNA expression of vascular endothelial growth factor in ischemic retinas of Macaca fascicularis. Invest Ophthalmol Vis Sci. 1996;37(7):1334-1340.

51. Pierce EA, Avery RL, Foley ED, Aiello LP, Smith LE. Vascular endothelial growth factor/vascular permeability factor expression in a mouse model of retinal NV. Proc Natl Acad Sci U S A. 1995;92(3) 905-909.

52. Stone J, Itin A, Alon T, et al. Development of retinal vasculature is mediated by hypoxia-induced vascular endothelial growth factor (VEGF) expression by neuroglia. $J$ Neurosci. 1995;15(7 Pt 1):4738-4747.

53. Aiello LP, Pierce EA, Foley ED, et al. Suppression of retinal NV in vivo by inhibition of vascular endothelial growth factor (VEGF) using soluble VEGF-receptor chimeric proteins. Proc Natl Acad Sci U S A 1995;92(23):10457-10461.

54. Alon T, Hemo I, Itin A, Pe'er J, Stone J, Keshet E. Vascular endothelial growth factor acts as a survival factor for newly formed retinal vessels and has implications for retinopathy of prematurity. Nat Med. 1995; 1(10):1024-1028.

55. Pierce EA, Foley ED, Smith LE. Regulation of vascular endothelial growth factor by oxygen in a model of retinopathy of prematurity. Arch Ophthalmol. 1996;114(10):1219-1228.

56. Sweigard JH, Yanai R, Gaissert P, et al. The alternative complement pathway regulates pathological angiogenesis in the retina. FASEB $J$. 2014;28(7):3171-3182.

57. Stalmans I, Ng YS, Rohan R, et al. Arteriolar and venular patterning in retinas of mice selectively expressing VEGF isoforms. J Clin Invest. 2002;109(3):327-336.

58. Ferrari G, Cook BD, Terushkin V, Pintucci G, Mignatti P. Transforming growth factor-beta 1 (TGF-beta1) induces angiogenesis through vascular endothelial growth factor (VEGF)-mediated apoptosis. $J$ Cell Physiol. 2009;219(2):449-458.
59. Chen J, Connor KM, Aderman CM, Smith LEH. Erythropoietin deficiency decreases vascular stability in mice. $J$ Clin Invest. 2008;118(2):526-533.

60. Lofqvist C, Willett KL, Aspegren O, et al. Quantification and localization of the IGF/insulin system expression in retinal blood vessels and neurons during oxygen-induced retinopathy in mice. Invest Ophthalmol Vis Sci. 2009;50(4):1831-1837.

61. Connor KM, SanGiovanni JP, Lofqvist C, et al. Increased dietary intake of omega-3-polyunsaturated fatty acids reduces pathological retinal angiogenesis. Nat Med. 2007;13(7):868-873.

62. Van Wijngaarden P, Coster DJ, Brereton HM, Gibbins IL, Williams KA. Strain-dependent differences in oxygen-induced retinopathy in the inbred rat. Invest Ophthalmol Vis Sci. 2005;46(4):1445-1452.

63. Rohan RM, Fernandez A, Udagawa T, Yuan J, D'Amato RJ. Genetic heterogeneity of angiogenesis in mice. FASEB J. 2000;14(7):871-876.

64. Chan CK, Pham LN, Chinn C, et al. Mouse strain-dependent heterogeneity of resting limbal vasculature. Invest Ophthalmol Vis Sci. 2004;45(2):441-447.

65. Walsh N, Bravo-Nuevo A, Geller S, Stone J. Resistance of photoreceptors in the $\mathrm{C} 57 \mathrm{BL} / 6-\mathrm{c} 2 \mathrm{~J}, \mathrm{C} 57 \mathrm{BL} / 6 \mathrm{~J}$, and BALB/cJ mouse strains to oxygen stress: evidence of an oxygen phenotype. Curr Eye Res. 2004;29(6):441-447.

66. Chan CK, Pham LN, Zhou J, Spee C, Ryan SJ, Hinton DR. Differential expression of pro- and antiangiogenic factors in mouse strain-dependent hypoxia-induced retinal NV. Lab Invest. 2005;85(6):721-733.

67. Vanhaesebrouck S, Daniels H, Moons L, Vanhole C, Carmeliet P, De Zegher F. Oxygen-induced retinopathy in mice: amplification by neonatal IGF-I deficit and attenuation by IGF-I administration. Pediatr Res. 2009;65(3):307-310.

68. Tan SM, Stefanovic N, Tan G, Wilkinson-Berka JL, de Haan JB. Lack of the antioxidant glutathione peroxidase-1 (GPx1) exacerbates retinopathy of prematurity in mice. Invest Ophthalmol Vis Sci. 2013;54(1):555-562.

69. Sears JE, Pietz J, Sonnie C, Dolcini D, Hoppe G. A change in oxygen supplementation can decrease the incidence of retinopathy of prematurity. Ophthalmology. 2009;116(3):513-518.

70. Stahl A, Chen J, Sapieha P, et al. Postnatal weight gain modifies severity and functional outcome of oxygen-induced proliferative retinopathy. Am J Pathol. 2010;177(6):2715-2723.

71. Bergman PB, Moravski CJ, Edmondson SR, et al. Expression of the IGF system in normal and diabetic transgenic (mRen-2)27 rat eye. Invest Ophthalmol Vis Sci. 2005;46(8):2708-2715.

72. Wilkinson-Berka JL, Wraight C, Werther G. The role of growth hormone, insulin-like growth factor and somatostatin in diabetic retinopathy. Curr Med Chem. 2006;13(27):3307-3317.

73. Hellström A, Hård A-L, Engström E, et al. Early weight gain predicts retinopathy in preterm infants: new, simple, efficient approach to screening. Pediatrics. 2009;123(4):e638-e645.

74. Löfqvist C, Andersson E, Sigurdsson J, et al. Longitudinal postnatal weight and insulin-like growth factor I measurements in the prediction of retinopathy of prematurity. Arch Ophthalmol. 2006;124(12):1711-1718.

75. Löfqvist C, Hansen-Pupp I, Andersson E, et al. Validation of a new retinopathy of prematurity screening method monitoring longitudinal postnatal weight and insulinlike growth factor I. Arch Ophthalmol. 2009; 127(5):622-627.

76. Holmes JM, Duffner LA. The effect of postnatal growth retardation on abnormal NV in the oxygen exposed neonatal rat. Curr Eye Res. 1996;15(4):403-409.

77. Ackerman B, Sherwonit E, Williams J. Reduced incidental light exposure: effect on the development of retinopathy of prematurity in low birth weight infants. Pediatrics. 1989;83(6):958-962.

78. Glass P, Avery GB, Subramanian KN, Keys MP, Sostek AM, Friendly DS Effect of bright light in the hospital nursery on the incidence of retinopathy of prematurity. N Engl J Med. 1985;313(7):401-404.

79. Wesolowski E, Smith LE. Effect of light on oxygen-induced retinopathy in the mouse. Invest Ophthalmol Vis Sci. 1994;35(1):112-119. 
80. Ricci B, Lepore D, Iossa M, Santo A, D'urso M, Maggiano N. Effect of light on oxygen-induced retinopathy in the rat model. Doc Ophthalmol. 1990;74(4):287-301.

81. Kremer I, Levitt A, Goldberg G, Wilunsky E, Merlob P, Nissenkorn I. The effect of light on oxygen-induced vasoproliferative retinopathy in newborn kittens. Invest Ophthalmol Vis Sci. 1992;33(5):1595-1598.

82. Dorfman AL, Joly S, Hardy P, Chemtob S, Lachapelle P. The effect of oxygen and light on the structure and function of the neonatal rat retina. Doc Ophthalmol. 2008;118(1):37-54.

83. Natoli R, Valter K, Barbosa M, et al. $670 \mathrm{~nm}$ photobiomodulation as a novel protection against retinopathy of prematurity: evidence from oxygen induced retinopathy models. PLoS One. 2013;8(8):e72135.

84. Emberson JR, Whincup PH, Walker M, Thomas M, Alberti KGMM. Biochemical measures in a population-based study: effect of fasting duration and time of day. Ann Clin Biochem. 2002;39(Pt 5):493-501.

85. Smith LE, Shen W, Perruzzi C, et al. Regulation of vascular endothelial growth factor-dependent retinal NV by insulin-like growth factor-1 receptor. Nat Med. 1999;5(12):1390-1395.

86. Higgins RD, Yu K, Sanders RJ, Nandgaonkar BN, Rotschild T, Rifkin DB. Diltiazem reduces retinal NV in a mouse model of oxygen induced retinopathy. Curr Eye Res. 1999;18(1):20-27.

87. Lange C, Ehlken C, Martin G, et al. Intravitreal injection of the heparin analog 5-amino-2-naphthalenesulfonate reduces retinal NV in mice. Exp Eye Res. 2007;85(3):323-327.

88. Ritter MR, Banin E, Moreno SK, Aguilar E, Dorrell MI, Friedlander M. Myeloid progenitors differentiate into microglia and promote vascular repair in a model of ischemic retinopathy. $J$ Clin Invest. 2006;116(12):3266-3276.

89. Banin E, Dorrell MI, Aguilar E, et al. T2-TrpRS inhibits preretinal $\mathrm{NV}$ and enhances physiological vascular regrowth in OIR as assessed by a new method of quantification. Invest Ophthalmol Vis Sci. 2006;47(5):2125-2134.

90. Penn JS, Gay CA. Computerized digital image analysis of retinal vessel density: application to normoxic and hyperoxic rearing of the newborn rat. Exp Eye Res. 1992;54(3):329-336.

91. Davies MH, Stempel AJ, Powers MR. MCP-1 deficiency delays regression of pathologic retinal NV in a model of ischemic retinopathy. Invest Ophthalmol Vis Sci. 2008;49(9):4195-4202.

92. Paques M, Guyomard JL, Simonutti M, et al. Panretinal, high-resolution color photography of the mouse fundus. Invest Ophthalmol Vis Sci. 2007;48(6):2769-2774.

93. Furtado JM, Davies MH, Choi D, et al. Imaging retinal vascular changes in the mouse model of oxygen-induced retinopathy. Transl Vis Sci Technol. 2012;1(2):5.

94. Mezu-Ndubuisi OJ, Teng P, Wanek J, et al. In vivo retinal vascular oxygen tension imaging and fluorescein angiography in the mouse model of oxygen-induced retinopathy. Invest Ophthalmol Vis Sci. 2013;54(10):6968-6972.

95. Ishikawa K, Yoshida S, Kadota K, et al. Gene expression profile of hyperoxic and hypoxic retinas in a mouse model of oxygen-induced retinopathy. Invest Ophthalmol Vis Sci. 2010;51(8):4307.

96. Zhu Y, Natoli R, Valter K, Stone J. Differential gene expression in mouse retina related to regional differences in vulnerability to hyperoxia. $\mathrm{Mol}$ Vis. 2010;16:740-755.

97. Miyamoto N, Mandai M, Takagi H, et al. Contrasting effect of estrogen on VEGF induction under different oxygen status and its role in murine ROP. Invest Ophthalmol Vis Sci. 2002;43(6):2007-2014.

98. Brothwood M, Wolke D, Gamsu H, Benson J, Cooper D. Prognosis of the very low birthweight baby in relation to gender. Arch Dis Child. 1986;61(6):559-564.
99. Phelps DL, Brown DR, Tung B, et al. 28-day survival rates of 6676 neonates with birth weights of 1250 grams or less. Pediatrics. 1991;87(1):7-17.

100. Palmer EA, Flynn JT, Hardy RJ, et al. Incidence and early course of retinopathy of prematurity. The Cryotherapy for Retinopathy of Prematurity Cooperative Group. Ophthalmology. 1991;98(11):1628-1640.

101. Yang MB, Donovan EF, Wagge JR. Race, gender, and clinical risk index for babies (CRIB) score as predictors of severe retinopathy of prematurity. JAAPOS. 2006;10(3):253-261.

102. Flower RW, McLeod DS, Lutty GA, Goldberg B, Wajer SD. Postnatal retinal vascular development of the puppy. Invest Ophthalmol Vis Sci. 1985;26(7):957-968.

103. Shabo AL, Maxwell DS. Insulin-induced immunogenic retinopathy resembling the retinitis proliferans of diabetes. Trans Sect Ophthalmol Am Acad Ophthalmol Otolaryngol. 1976;81(3 Pt 1):497-508.

104. Langer HF, Chung K-J, Orlova VV, et al. Complement-mediated inhibition of NV reveals a point of convergence between innate immunity and angiogenesis. Blood. 2010;116(22):4395-4403.

105. Ishida S, Yamashiro K, Usui T, et al. Leukocytes mediate retinal vascular remodeling during development and vaso-obliteration in disease. Nat Med. 2003;9(6):781-788.

106. Vessey KA, Wilkinson-Berka JL, Fletcher EL. Characterization of retinal function and glial cell response in a mouse model of oxygeninduced retinopathy. J Comp Neurol. 2011;519(3):506-527.

107. Dorrell MI, Aguilar E, Jacobson R, et al. Maintaining retinal astrocytes normalizes revascularization and prevents vascular pathology associated with oxygen-induced retinopathy. Glia. 2010;58(1):43-54.

108. Liu X, Wang D, Liu Y, et al. Neuronal-driven angiogenesis: role of $\mathrm{NGF}$ in retinal NV in an oxygen-induced retinopathy model. Invest Ophthalmol Vis Sci. 2010;51(7):3749-3757.

109. Saito Y, Geisen P, Uppal A, Hartnett ME. Inhibition of NAD(P)H oxidase reduces apoptosis and avascular retina in an animal model of retinopathy of prematurity. Mol Vis. 2007;13:840-853.

110. Wilkinson-Berka JL, Deliyanti D, Rana I, et al. NADPH oxidase, NOX1, mediates vascular injury in ischemic retinopathy. Antioxid Redox Signal. 2014;20(17):2726-2740.

111. Wang H, Zhang SX, Hartnett ME. Signaling pathways triggered by oxidative stress that mediate features of severe retinopathy of prematurity. JAMA Ophthalmol. 2013;131(1):80-85.

112. Li S-Y, Fu ZJ, Lo ACY. Hypoxia-induced oxidative stress in ischemic retinopathy. Oxid Med Cell Longev. 2012;2012:426769.

113. Tan SM, Deliyanti D, Figgett WA, Talia DM, de Haan JB, WilkinsonBerka JL. Ebselen by modulating oxidative stress improves hypoxiainduced macroglial Müller cell and vascular injury in the retina. Exp Eye Res. 2015;136:1-8.

114. Ristori C, Filippi L, Dal Monte M, et al. Role of the adrenergic system in a mouse model of oxygen-induced retinopathy: antiangiogenic effects of beta-adrenoreceptor blockade. Invest Ophthalmol Vis Sci. 2011;52(1):155-170.

115. Martini D, Monte MD, Ristori C, et al. Antiangiogenic effects of $\beta 2$-adrenergic receptor blockade in a mouse model of oxygen-induced retinopathy. J Neurochem. 2011;119(6):1317-1329.

116. Grossniklaus HE, Kang SJ, Berglin L. Animal models of choroidal and retinal NV. Prog Retin Eye Res. 2010;29(6):500-519.

117. Cao Z, Jensen LD, Rouhi P, et al. Hypoxia-induced retinopathy model in adult zebrafish. Nat Protoc. 2010;5(12):1903-1910.

118. Cao R, Jensen LDE, Söll I, Hauptmann G, Cao Y. Hypoxia-induced retinal angiogenesis in zebrafish as a model to study retinopathy. PLoS One. 2008;3(7):e2748. 
Eye and Brain

\section{Publish your work in this journal}

Eye and Brain is an international, peer-reviewed, open access journal focusing on clinical and experimental research in the field of neuroophthalmology. All aspects of patient care are addressed within the journal as well as basic research. Papers covering original research, basic science, clinical and epidemiological studies, reviews and evaluations,

Submit your manuscript here: http://www.dovepress.com/eye-and-brain-journal

\section{Dovepress}

guidelines, expert opinion and commentary, case reports and extended reports are welcome. The manuscript management system is completely online and includes a very quick and fair peer-review system, which is all easy to use. Visit http://www.dovepress.com/testimonials.php to read real quotes from published authors. 